\title{
Composition and Crystal Orientation Mapping of nano-scale multi-phase Rapid Solidification Microstructures in hypo-eutectic Al-Cu Alloy Thin Films
}

Jörg M.K. Wiezorek ${ }^{1}$, Can Liu ${ }^{1}$, Sahar Farjami ${ }^{1}$, Kai W. Zweiacker ${ }^{1,3}$, Joseph T. Mckeown ${ }^{2}$ and Geoffrey H. Campbell ${ }^{2}$

1. Department of Mechanical Engineering and Materials Science, Swanson School of Engineering, 636 Benedum Hall, 3600 O'Hara Street, University of Pittsburgh, Pittsburgh, PA 15261, USA.

2. Materials Science Division, Physical and Life Science Directorate, Lawrence Livermore National Laboratory, 7000 East Avenue, Livermore, CA, 94551, USA

3. Now at EMPA, Swiss Federal Laboratories for Materials Science and Technology Überlandstrasse 129, CH-8600 Dübendorf, Switzerland.

Recent nano-scale spatio-temporal resolution in situ transmission electron microscopy (TEM) studies of the rapid solidification (RS) of elemental $\mathrm{Al}$ and hypo-eutectic $\mathrm{Al}-\mathrm{Cu}$ alloys after pulsed laser melting permitted measurements of the evolution of crystal growth rates and correlation with crystal growth mode changes responsible for the microstructure formation under non-equilibrium conditions [1-4]. The morphological characteristics of the multi-phase RS microstructures in the electron transparent thin film $\mathrm{Al}-\mathrm{Cu}$ alloys were identical to those reported for equivalent composition bulk alloys [2-6]. Here we combine precession electron diffraction assisted automated crystal orientation mapping (PED-ACOM) with scanning (TEM) (STEM) imaging and energy dispersive X-ray spectroscopy composition mapping (EDXSM) to elaborate constitutional and crystallographic aspects of the development of multi-phase RS microstructures in hypo-eutectic $\mathrm{Al}-\mathrm{Cu}$ alloys with $4 \mathrm{at} \%$ to $12 \mathrm{at} \% \mathrm{Cu}$.

Fig. $1 \mathrm{a}$ and $1 \mathrm{~b}$ illustrate the refined scale and distinct morphology of the RS microstructures of hypoeutectic $\mathrm{Al}-\mathrm{Cu}$ with the heat affected zone (HAZ, Zone 1), a narrow transition region with elongated $\alpha$-Al grains (Zone 2 ), the RS crystal growth region with columnar morphology cellular twophase growth (Zone 3a), and the banded-morphology growth region (Zone 3b) [2,6]. The formation of these different microstructural zones is related to the changes in RS interface velocity after pulsed laser induced melting (e.g. Fig. 1c). After incubation periods that last from $\sim 5 \mu$ s to $\sim 25 \mu$ s (increasing with $\mathrm{Cu} \%$ ) the directional RS crystal growth forms the Zone 2, Zone $3 \mathrm{a}$ and Zone $3 \mathrm{~b}$ multiphase microstructures at crystal growth rates of $0.1 \mathrm{~m} / \mathrm{s}$ to $\sim 2.0 \mathrm{~m} / \mathrm{s}$ (e.g. Fig. 1c for Al-4Cu). RS front velocity changes profoundly affect the coupled growth of the $\alpha$-Al (dark, Fig. 1b) and $\mathrm{Al}_{2} \mathrm{Cu}$ (bright, Fig. 1b). Fig. 2 shows STEM DF-imaging and EDXSM data obtained for Zones 1 and 2 in Al-11Cu. For this alloy the $\alpha$-Al grains in Zone 1 contained 3.6at\%Cu (e.g. circles, Fig. 2a), which is about the same as for the e-beam deposited films prior to $\mathrm{RS}$ [6]. The $\mathrm{Al}_{2} \mathrm{Cu}$ phase in the triple junction of the continuous $\mathrm{Cu}$ enriched network in Zone 1 and Zone 2 (TJ in Fig. 2a) contained on average $31.5 \mathrm{at} \% \mathrm{Cu}$. Notably, the cellular growth zone (Zone 3a) exhibited discontinuous fine-scale copper enriched metastable $\mathrm{Al}_{2} \mathrm{Cu}\left(\theta^{\prime}\right)$ phase within the supersaturated $\alpha$-Al matrix grains, while the $\mathrm{Cu}$-enriched continuous network in Zone 1 and Zone 2 contained $\theta-\mathrm{Al}_{2} \mathrm{Cu}$ phase (Figs. 1 and 2) [2-4]. Under equilibrium conditions hypoeutectic $\mathrm{Al}-\mathrm{Cu}$ alloys contain the face-centered cubic $\alpha$-Al matrix $(\leq 2.6$ at $\% \mathrm{Cu})$ and tetragonal $\theta-\mathrm{Al}_{2} \mathrm{Cu}$ $(\sim 33 \mathrm{at} \% \mathrm{Cu})$ phase as primary pro-eutectic and secondary lamellar eutectic microstructural constituents. The PEDACOM and EDXSM analyses of the hypo-eutectic Al-Cu alloys showed deviations of the phase fractions and the compositions from those of the as-deposited stated and those predicted by the equilibrium phase diagram for $\alpha-\mathrm{Al}$ and $\mathrm{Al}_{2} \mathrm{Cu}$ for the $\mathrm{RS}$ microstructure Zones 2, 3a and 3b. EDXSM consistently showed severe composition gradients for $\alpha$-Al in the narrow Zone 2 transition region 
$(\leq 1 \mu \mathrm{m})$ from slow solidification rates below $0.1 \mathrm{~m} / \mathrm{s}$ to RS velocities $\geq 0.5 \mathrm{~m} / \mathrm{s}$, while the supersaturated $\alpha$-Al phase compositions remained constant in Zone $3 \mathrm{a}$ and reached alloy composition for the singlephase crystallization at transition to Zone $3 \mathrm{~b}$. The discontinuous morphology of the $\mathrm{Al}_{2} \mathrm{Cu}\left(\theta^{\prime}\right)$ phase in the Zone $3 \mathrm{a}$ implies continuous growth of the matrix Al crystal and repeated nucleation and growth for the minority $\mathrm{Al}_{2} \mathrm{Cu}$ crystals during $\mathrm{RS}$ at interface velocities in excess of $0.5 \mathrm{~m} / \mathrm{s}[3,4]$. Finally, two different crystallographic orientation relationships between the $\alpha$-Al matrix and the $\mathrm{Al}_{2} \mathrm{Cu}\left(\theta^{\prime}\right)$ phase that facilitate coherent interphase interface formation have been identified by PEDACOM.

[1] K. Zweiacker et al, Journal of Applied Physics 120 (2016) p.055106.

[2] J.T. McKeown et al, Acta Materialia 65 (2014), p. 56.

[3] J.T. McKeown et al, JOM 68 (2016), p. 985.

[4] K.W. Zweiacker et al. Microsc. Microanal. 21 (S3) (2015) p.1465.

[5] W. Kurz and P. Gilgien, Materials Science and Engineering A 178(1994), p. 171.

[6] The authors acknowledge funding from the National Science Foundation, Grants NSF-1105757 and

NSF-1607922, and the U.S. Department of Energy, Office of Basic Energy Sciences, Division of Materials Sciences and Engineering for FWP SCW0974 by Lawrence Livermore National Laboratory under Contract DE-AC52-07NA27344.
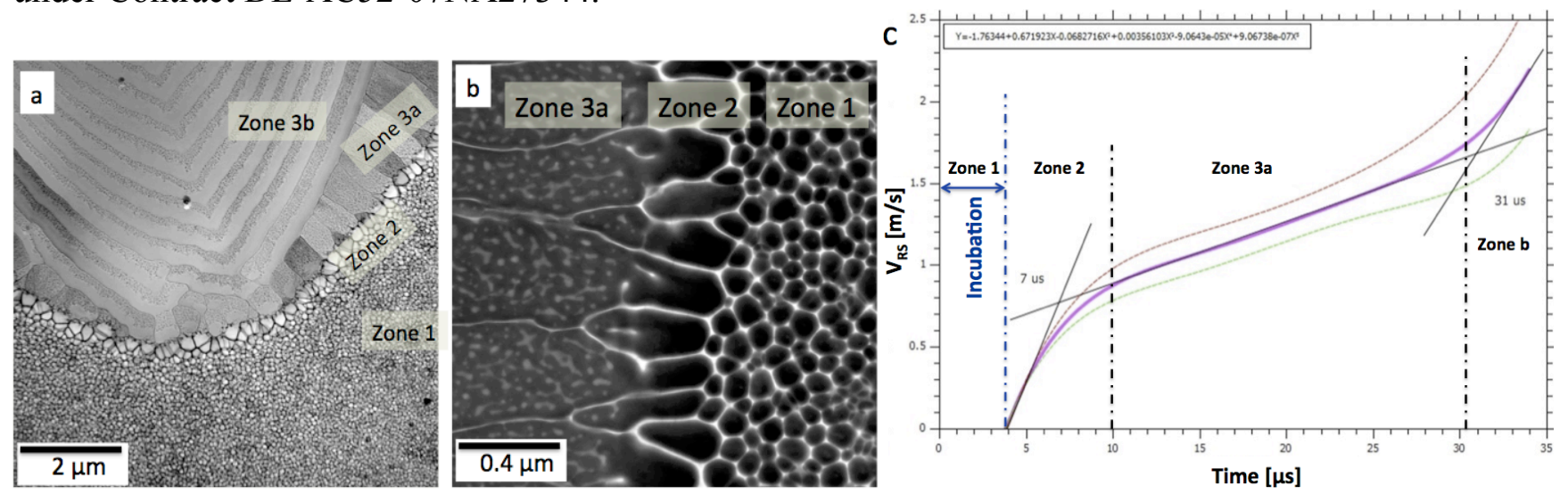

Figure 1. The RS microstructure zones, a) BF-, b) HAADF STEM images for Al-9Cu and c) RS interface velocity evolution for hypo-eutectic Al-4Cu after PL melting for Al-4Cu.

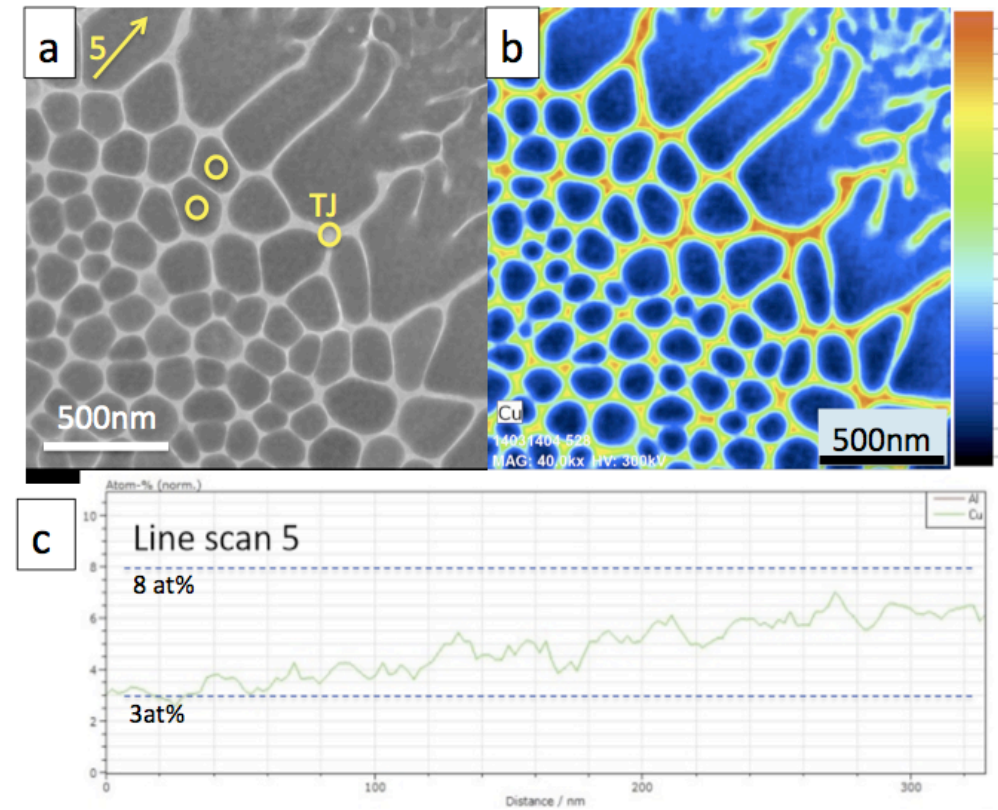

Figure 2. a) HAADF STEM image for Al-11Cu alloy with locations of example line scan for Zone 2 and compositions of $\alpha$-Al grains and triple junctions (TJ) in the continuous network of $\mathrm{Cu}$-enriched phase in Zone 1, b) $\mathrm{Cu} \%$ heat map for $\mathrm{Cu} \% \leq 35 \mathrm{at} \%$, c) example of $350 \mathrm{~nm}$ length $\mathrm{Cu} \%$ line scan for elongated $\alpha$-Al grain in Zone 2 (marked in a)) with $\mathrm{Cu} \%$ increasing from $3 \mathrm{at} \%$ to $6 \mathrm{at} \%$. 